\title{
Sección Informativa
}

\section{CONGRESO COLOMBIANO DE OBSTETRICIA Y GINECOLOGIA}

Bogotá, D. E. Diciembre 5,6,7 de 1979

Información e Inscripciones: Edificio Academia Nacional de Medicina

Oficina No. 602. BOGOTA.

\section{CONGRESO MUNDIAL DE GINECO- LOGIA Y OBSTETRICIA}

Tokio. Japón. Octubre 25-31 de 1979

Información: Simul International Inc. i,8,10. Akasaka. Minato-ku Tokio. 107, Japan.

X CONGRESO MUNDIAL DE GINECOLOGIA Y OBSTETRICIA (aviso anticipado),

San Francisco. California. Octubre de 1982.

Información: (a partir de diciembre de 1979 en FECOLSOG. Bogotá)

\section{CURSOS}

Simposio Internacional "Fisiopatología Endocrina del Ovario"

Rosario. (Argentina) del 23 al 26 de mayo de 1979

Temas: 1. Progresos en anatomía microscópica y ultramicros-

2. Desarrollo morfológico del ovario.

3. Fisiología del Ovario.

4. Interacciones endocrinas del Ovario.

5. Progresos en el estudio de la función del ovario

6. Aspectos clínicos de los diferentes grados de insuficiencia ovárica

7. Fallo ovárico severo

8. Inducción de la ovulación Información: Dr. Rafael Ruíz Pineda. ta Fe 1798/2000 Rosario (Argentina)

Curso de Citología Ginecológica Medellín. (Colombia) Enero 25-26-27 de 1979.

Curso dictado por los Drs. Leo y Lencioni (Argentina). Presidente de la Sociedad Latinoamericana de Citología. Marcos Peluffo (Argentina). Lucrecia Illescas de Peluffo (Argentina). Armando Santamaría (Bogotá). Francisco Cabanzo (Bogotá). Jorge Medina (Bogotá) Nubia Aristizábal (Cáli).

Información: Dr. Fernando Cardona. Calle 56 No. 43-17 Medellín - Colombia. 